\title{
THE NEAR FIELD IN THE MIXING OF A THREE-DIMENSIONAL INCLINED POLLUTANT JET WITH A CROSSFLOW
}

\author{
Sä̈ N. Mahjoub, ${ }^{1, *}$ S. Habli, ${ }^{1}$ H. Bournot, ${ }^{2}$ E G. Le Palec ${ }^{2}$ \\ ${ }^{1}$ Institut Préparatoire aux Etudes d'Ingénieurs de Monastir, Monastir, Tunisie \\ ${ }^{2}$ IUSTI, UMR 6595, Technopôle de Château-Gombert, Marseille, France
}

\begin{abstract}
An experimental investigation was carried out to study the structure of the flow field resulting from the interaction of an inclined pollutant jet with a crossflow. The study of the flow field was conducted in a wind tunnel test by means of particle image velocimetry (PIV) system. As the jet was discharged with a variable angle, the resulting flow has been found to be quite complex owing to its three-dimensional nature and the interaction between several flow regions. Results showed the dependence of the emerging jet flow structure on its ratio velocity and the Reynolds number. Extensive wind tunnel experimental results are presented; they concern the Kelvin-Helmholtz vortical structures and the effect of the velocity ratio $v_{0} / u_{\infty}$ on the interacting zone. A three-dimensional numerical model with a second-order turbulent model (RSM) and a nonuniform grid system is used to examine the behavior of the emerging jet. The comparison of the numerical and experimental results gives satisfactory agreement.
\end{abstract}

KEY WORDS: experimental study, numerical simulation, inclined jet, PIV, turbulent flow, recirculation zone

\section{INTRODUCTION}

Interactions between cool/hot jets with crossflows are highly interesting due to their wide presence in several industrial applications. Active flow control, aircraft performance and stability, mixing augmentation, film and effusion cooling, etc., are some of these applications. Perhaps the most familiar example in everyday life would be smoke rising from a chimney into a crosswind. This comes after film cooling, which is a technique extensively used in turbomachinery (Azzi at al., 2001). The use of film cooling has particularly increased as a solution for the present trend towards the implementation of higher inlet temperatures for the generation of higher power. In addition to their wide application, jets in crossflow are interesting due to the complexity of the flowfield they generate. In fact once discharged, the jet is rapidly deflected in the direction of the crossflow, giving rise to a kidney-shaped cross section, dominated by a counterrotating vortex pair (CVP). As the potential core of the jet ends as a cone, a mixing layer develops at the periphery, slightly bent in the direction of the crossflow.

The great practical relevance of the generated mechanisms inspired several experimental, numerical, and theoretical studies. After the interaction between the inclined jet and the mainstream, one of the most experimentally highlighted features is the formation of the counterrotating vortex pair, its origin, and the mechanism of its formation. Taylor et al. (1982) are some of the pioneers that dealt with this feature, the CVP. Consideration was given to a turbulent jet discharged into a turbulent subsonic crosswind, under a variable inclination $\left(15^{\circ}-90^{\circ}\right)$ and momentum ratio. The results were given in terms of the jet paths (based on the maximum total pressure), deflection and penetration, the lift loss, and the pressure distribution. A decrease in the jet's inclination (from the position normal to the crosswind) reduced all these features together with a downstream move of the pressure center. In Broadwell and Breidenthal's paper (1984), the CVP vortices were suggested to rise from the impulse associ- 


\section{NOMENCLATURE}

$d \quad$ minor diameter of the jet nozzle, $\mathrm{m}$

$D \quad$ major diameter of the jet nozzle, $\mathrm{m}$

$f \quad$ mass fraction

$g \quad$ gravitational acceleration, $\mathrm{m} / \mathrm{s}^{2}$

$G_{k} \quad$ term of production due to buoyancy forces, $\mathrm{kg} /\left(\mathrm{m} \cdot \mathrm{s}^{3}\right)$

$H_{T} \quad$ wind tunnel height, $\mathrm{m}$

$k \quad$ kinetic energy of turbulence, $\mathrm{m}^{2} / \mathrm{s}^{2}$

$n \quad$ the normal on the considered surface, $\mathrm{m}$

$P_{k} \quad$ term of production due to the mean gradients, $\mathrm{kg} /\left(\mathrm{m} \cdot \mathrm{s}^{3}\right)$

$R \quad$ velocity ratio $v_{0} / u_{\infty}$

Re Reynolds number $v_{0} d / v$

$\operatorname{Re}_{F} \quad$ Reynolds number $u_{\infty} H_{T} / v$

$S_{i j} \quad$ mean strain rate

$T$ temperature

$u_{\infty} \quad$ crossflow velocity, $\mathrm{m} / \mathrm{s}$

$v \quad$ injection velocity, $\mathrm{m} / \mathrm{s}$

$\overline{u_{i}^{\prime \prime} u_{j}^{\prime \prime}} \quad$ Reynolds stress, $\mathrm{m}^{2} / \mathrm{s}^{2}$

$u_{i}, u_{j} \quad$ velocity components along the $i$ and $j$ directions $\begin{array}{ll}u, v, w & \text { velocity components along } \\ & x, y, \text { and } z \text { directions, } \mathrm{m} / \mathrm{s}\end{array}$

\section{Greek Symbols}

$\rho$ density, $\mathrm{kg} / \mathrm{m}^{3}$

$\beta \quad$ thermal expansion coefficient

$\varepsilon$ dissipation rate of the turbulent

kinetic energy

$\mu \quad$ kinetic viscosityb, $\mathrm{kg} /(\mathrm{m} \cdot \mathrm{s})$

$\mu_{t} \quad$ turbulent (or eddy) viscosity, $\mathrm{kg} /(\mathrm{m} \cdot \mathrm{s}$ )

$\mu_{t} \quad$ turbulent (or eddy) viscosity, $\mathrm{kg} /(\mathrm{m} \cdot \mathrm{s}$ )

$\delta_{i j} \quad$ Kronecker symbol (=1 if $i=j$ and 0 if $i \neq j$ )

\section{Subscripts}

$\infty \quad$ conditions in crossflow

0 exit section of the jet

\section{Superscripts \\ - $\quad$ Reynolds average \\ $\sim \quad$ Favre average}

ated with the jet. It is then a global feature of the far field, in which the CVP are convected downstream by the crossflow. More detailed experimental studies, concentrated on the near-field, suggested that the CVP vortices are generated by the vortex sheet emanating from the jet nozzles or pipes (Moussa, et al., 1977; Andreopoulos and Rodi, 1984; Fric, 1990; Kelso et al., 1996). Moussa et al. (1977) focused on the near-field, defined as the region of the flow that stretches from the jet exit to a distance of a few diameters downstream of this exit. The confrontation between the discharged jet and the surrounding mainstream, in this region, brings three main adjustments on the jet's progression, which are closely dependent on the geometry of jet exit boundaries. A pair of bound vortices embedded within the lee side of the jet boundary develops and helps accelerate the development of the turbulent and mean vorticity. A vortex-shedding system then takes place and is accompanied by an increase in the entrainment rate of the mainstream into the jet. In addition to these observations, calculations of mass, momentum, and vorticity fluxes have been made and proved that the vorticity flux is at the origin of the rapid stretching and tilting of the vorticity vector field in the near-field region.

The following papers did not focus on a specific feature of the resulting field of a jet interacting with a crossflow. Attention was rather paid to the overall developed dynamic structures, and their evolution and growth either under constant or variable parameters. Chu (1985), for example, described the jet's motion in a moving oblique coordinate system in the case of an oblique turbulent jet in a uniform crossflow by means of a line-impulse model.

The experiments of Lee et al. (1994) highlighted the overall flow features induced by a normal and streamwise $35^{\circ}$ inclined jets, discharged into a turbulent crossflow boundary layer under a variable velocity ratio. The examined features include the turbulent activity enhanced by small velocity ratios in addition to the bound vortices and the downstream complex three-dimensional flow, which develop simultaneously regardless of the injection ratio and the initial inclination of the jets.

The numerical investigations of Jansson and Davidson (1994) focused on the film cooling effectiveness and the 
velocity fields in an attempt to evaluate the efficiency of modeling of the finite volume calculations on a "jet in crossflow" configuration. The model efficiency was established with reference to two different turbulence models, namely the standard $k-\varepsilon$ model and a full Reynolds stress model (RSM) in addition to hot wire measurements. Near the walls, the viscous effects on turbulence were determined by means of a one-equation model, elaborated by Norris and Reynolds (1975) together with a mixing layer approach. A similar modeling evaluation was carried out by Donohue et al. (1994), but on the efficiency of experimental iodine fluorescence-based data, over a swept ramp injection. A satisfying level of agreement was observed, though with some deviations, generally in the range of 5\%, but larger such as in the base of the ramp. This evaluating investigation could also reveal asymmetry of the resulting flowfield and ramp-generated vortices, in addition to the turning and mixing of the jet plume.

Laser Doppler velocimetry measurements were used by Santiago and Dutton (1997) and Zhang and Collins (1997) for the examination of inclined jets in crossflow. While the jet was circular and discharged within a supersonic crossflow within the first work (Santiago and Dutton, 1997) it was rectangular, pitched, skewed, and discharged into a low speed wind tunnel crossflow within the second paper (Zhang and Collins, 1997). Attention was given to the developed vortical structures in both works with a further exploration of the mean and fluctuating quantities within the first one and comparison of the developed vortices against the circular jet case in the second.

Further experimental techniques were used for the exploration of inclined jets in crossflow, such as the plane laser light illumination (Nakabe et al., 1998), the planar laser-induced fluorescence (PLIF) (Rivero et al., 2001), hot wire anemometry (Milanovic and Zaman, 2003), etc. Nakabe et al. (1998) combined laser light illumination, fluorescent dyes and color-to-temperature conversion of thermo-chromatic liquid crystal sheets to characterize the velocity field and the generated heat transfer. Rivero et al. (2001) used the PLIF visualizations to identify and provide plausible origin to the established vortical structures, namely the CVP and upright vortices, folded vortex rings, horseshoe vortices, and the newly observed handle-type structures. As to Milanovic and Zaman (2003), they focused rather on the velocity field and the generated turbulent stresses. The experiments carried out by Taslim and Ugarte (2004) paid attention to the impact of the jets' inclination, the presence or not of a surrounding crossflow, the pressure ratio and the shape of the discharging hole on the coolant blowing rate, and consequently on the cooling effectiveness.

In the last decade, several numerical studies, based on Large Eddy Simulations (LES) (Wegner et al., 2004; Guo et al., 2006), were carried out on inclined jets in crossflow. The simulations of Wegner et al. (2004) were validated against the experimental data of Andreopoulos (1982) and Andreopoulos and Rodi (1984). Wegner et al. (2004) checked whether active or passive means, including the jets' inclination, may help in controlling and enhancing the resulting mixing process, its vortical structures, and the induced secondary motion. Guo et al. (2006) investigated the cooling performance of the discharged jets, the turbulent flow structures they produce, and the induced vortex dynamics. Particular attention was dedicated to the counter-rotating vortex pair (CVP), its first appearance, magnitude, and location under a variable injection ratio. In a prior paper, Guo et al. (2004) used SIPLEC algorithm, body-fitted coordinates, the wall function method, and the RNG $k-\varepsilon$ turbulence model for the modeling of the same configuration, namely a variably inclined jet in crossflow responding to different injection ratios. Once validated against experimental measurements, the model could describe the jet trajectories, define the separation events in the lee of the jet exit and allowed analyzing the different developing mechanisms. The numerical threedimensional simulation of Yang and Wang (2005) added the impingement mechanism within the jet in crossflow model to assess the generated heat transfer. The analysis is based upon the $k-\varepsilon$ turbulence model and its associate wall function in addition to a control-volume-based finite-difference method with power-law scheme.

Jia et al. (2005) combined numerical simulations and laser Doppler velocimetry (LDV) experiments on a jet variably inclined and sent at different injection ratios in an attempt to optimize the film cooling performance of the discharged jet. The cooling performance was determined in terms of the induced recirculation bubble. Jessen et al. (2007) assessed this cooling performance experimentally by means of particle image velocimetry (PIV) measurements, in terms of the developed turbulent flow structures and vortex dynamics, under variable injection, density, and mass fraction ratios. A higher mass flux ratio enlarges the size of the recirculation region, leading to a more pronounced entrainment of hot outer fluid into the wake of the jet and a higher density ratio enhances the lateral spreading of the coolant downstream, which is crucial for the cooling efficiency.

The present paper checks some previous results through comparisons, and tries to extend them. Based 
upon PIV-based experiments, our investigation assesses the impact of both the jet inclination and the velocity ratio on the efficiency of mixing of a variably inclined jet within a uniform crossflow. The measured data, in terms of velocity fields, are combined with three-dimensional numerical results based upon the RSM (Reynolds stress model) turbulence closure model and a nonuniform grid system, to account for the jet's inclination impact on the generated field. Once validated, the effect of the jet's inclination impact is generalized in order to investigate both the thermal field and the dispersion process of the pollutants embedded within the discharged jet. Similar results are likely to offer engineers an extra consideration for practical applications of an inclined jet in crossflow, particularly for applications with direct impact on environmental issues.

\section{PRESENTATION OF THE PROBLEM}

Intuitively simple, the topology of an inclined jet injected into a transverse flow is in fact very complex. In fact, after its injection, the jet fluid is deflected in the direction of the crossflow as its cross section quickly assumes a kidney shape, dominated by a pair of counter-rotating vortices (Fig. 1, II). A mixing layer develops on the periphery of the jet as the potential core of the jet ends as a cone, slightly bent in the direction of the crossflow. The various swirling structures generated by the interaction of a jet and a transverse flow are represented in Fig. 1.

Swirls of the boundary layer of the jet develop and are accompanied by the following specific structures:

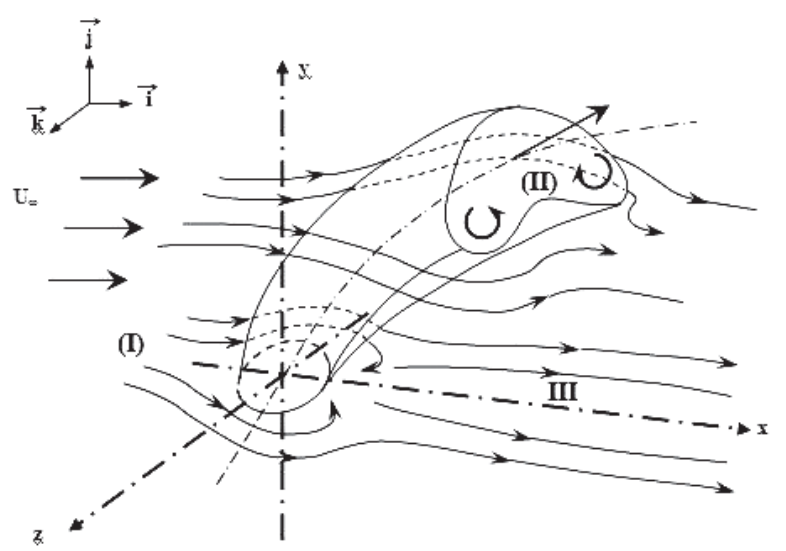

FIG. 1: Scheme of the existing swirling structures of the interaction of an inclined jet and transverse crossflow.
- Horseshoe swirls (along the wall); they are similar to that which appears near the junction of a wing and a plane plate (I)

- Strongly three-dimensional wake swirls resembling a Von Karman street downstream of a circular cylinder (III) (from jet to wall). The physical analogy between these two phenomena is only very partial because, in addition to the curvature of the jet, there is no production of vorticity on the boundary between the jet and the transverse flow, contrary to the wall of a cylinder where the condition of no-slip implies a production of vorticity.

- The contrarotating vortices pair (II), around which the flow organizes. The origin of this pair of contrarotating swirls (CVP for "counter-rotating vortex pair") was the subject of many experimental (Kelso and Lim, 1996) as well as numerical (Cortelezzi and Karagozian, 2001) investigations. It was thus established that the CVP appeared under the combined effects of the crumpling, the slope, and finally of the rolling up of the natural swirling structures of the jet under the effect of the transverse flow.

- Swirls of the sheared layer all around the jet.

Another dividing of the flow field is available in the literature and it depends on the nature of the fluid in the entire domain: near-field mixing, which is usually controlled entirely by the initial conditions, such as geometry of the jet, exit velocity, initial density difference, turbulence intensity, and velocity distribution in the supply pipe (Fischer et al., 1979). It is a region of rapid mixing and dilution caused by turbulence generated by the discharge.

Zone of flow establishment: This region refers to the distance immediately after the end of the nozzle, where a flow transition occurs between the efflux velocity profiles seen in the discharge pipe to the Gaussian profile exhibited in the jet (Jirka et al., 1992). The fluid is "unaware" that it is suddenly in a different environment, and it takes some distance to adjust to the new flow characteristics.

Far-field mixing occurs beyond the near-field mixing process, where dynamic source characteristics lose their importance and ambient properties begin to control trajectory, advection, and mixing (Roberts, 1996).

\section{EXPERIMENTAL SETUP}

Measurements were carried out in a wind tunnel. This tunnel had a test section with a speed range of about $0-16$ 
$\mathrm{m} \cdot \mathrm{s}^{-1}$. The experiments were performed in an open circuit discharging to atmosphere outside the laboratory and with a parallel-sided, closed working section $3 \mathrm{~m}$ in length and $0.2 \mathrm{~m} \times 0.3 \mathrm{~m}$ in cross section. For optical access, one side is made of Pyrex; the interior of the wind tunnel is painted black to reduce reflections. In all experiments, the airflow is seeded with oil droplets of approximately $0.8 \mu \mathrm{m}$ diameter, and introduced at the ambient temperature into the tunnel nozzle. The jet was produced by means of a smooth pipe and $10 \mathrm{~mm}$ diameter $(d)$ through which air at constant temperature was discharged. The pipe was positioned at the upstream end $(20 \mathrm{~cm}$ from the tunnel nozzle) and along the centerline of the tunnel oriented at an angle of $60^{\circ}$ to the crossflow. The jet is seeded with approximately $1 \mu \mathrm{m}$ diameter glycerin particles (seeding density $\approx 30$ particles $\mathrm{ml}^{-1}$ of pure jet fluid). The turbulence intensity level of the crossflow was less than $0.2 \%$. The Reynolds numbers for flow at the entry of the wind tunnel $\left(\operatorname{Re}_{F}=u_{\infty} H_{T} / v\right)$ were in the range $2.7 \times 10^{4}-2.2 \times 10^{5}$ while within the jet the Reynolds numbers $\left(\operatorname{Re}=v_{0} d / v\right)$ were in the range $1.1 \times 10^{3}$ to $6 \times$ $10^{3}$.

The particle image velocimetry system (Fig. 2) is based upon a TSI Power View system, including a $50 \mathrm{~mJ}$ dual YAG laser which produces two plan luminous pulses, the duration of one ranging from $5 \times 10^{-9}$ to $10^{-8}$ seconds, a Power View 4M high resolution cross-correlation camera ( $2 \mathrm{k} \times 2 \mathrm{k}$ resolution, 12 bits), a synchronizer, and "Insight" Windows-based software for acquisition, processing, and post-processing. This software allows the synchronization of pulsations according to the observed phenomena, and the adjustment of the time step between two images. This time step was $70 \mu \mathrm{s}$. In order to avoid errors, the velocity vectors were calibrated at $130 \mu \mathrm{m} /$ pixel

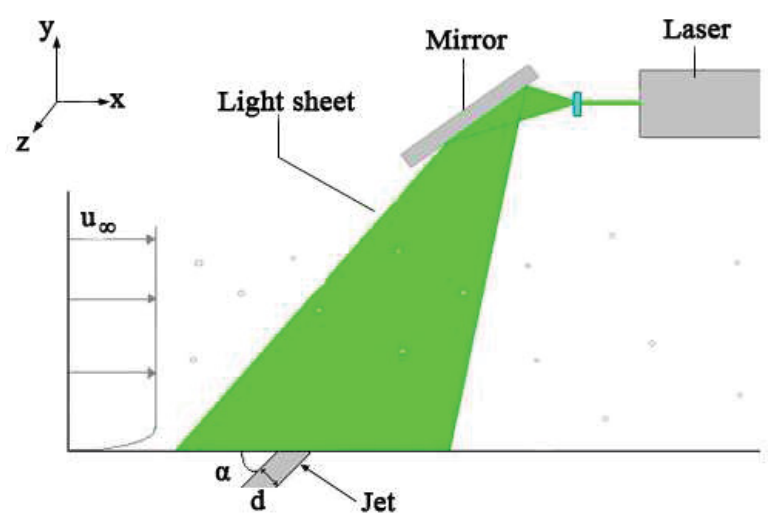

FIG. 2: Experimental setup: PIV. and limited to the representation of the velocity field in the regions where the luminance was strong enough.

Laser pulses from a neodymium-doped yttrium aluminum garnet (ND-YAG) laser were expanded to form a thin light sheet using a cylindrical lens. The light sheet was brought from the top of the wind tunnel using a $45^{\circ}$ mirror. Because of this arrangement, a shadow area upstream from the pipe was created where it was impossible to take measurements.

The light scattered from the seeded particles was captured and the resulted scattering signal is collected by an interline transfer CCD camera. The camera was oriented perpendicular to the plane of the sheet. The frame transfer capability of this camera allows each of the two closely spaced laser sheet pulses to be captured in a separate image. The collected images were transferred to the control computer. A target image was used to provide accurate scaling of the flow.

However, after the vector field is initially processed, filters are applied to the data to eliminate spurious vectors, and these vectors are linearly interpolated. Then the vector field is reprocessed. This permits the use of a crosscorrelation PIV algorithm (Hasselbrink, 1999), which eliminates directional ambiguity and yields improved resolution over single-image autocorrelation techniques. In this way, the in-plane loss-of-correlation is minimized, and therefore the chance of valid vector detection is maximized.

Once an ensemble of instantaneous vector fields has been obtained from a set of images, several postprocessing calculations are made. Raw data are cast from pixel units into physical coordinates, and turbulence statistics and derived quantities such as vorticity and strain rate are calculated, using custom software. The final fields were averages performed with 200 successive acquisitions.

For each point, experimental uncertainties are estimated with the following criterion:

$$
\frac{V_{\max }-V_{\min }}{V_{\mathrm{av}}}
$$

where $V_{\max }, V_{\min }$, and $V_{\text {av }}$, respectively are the maximum velocity, the minimum velocity, and the average velocity measured during all the process. The uncertainties were about 5\%. The estimates were inferred from estimated inaccuracies in the calibration data and from the observed scatter in the measurements.

The velocity profile of the main flow was measured with a hot wire anemometer together with a high precision electronic pressure transducer. With the help of an on-line 
micropressure calibration system, the uncertainty in the free-stream velocity was estimated to be as large as 3\% of a reading.

The origin of the wake coordinate system is fixed on the axis of the pipe. The $x$ axis is directed downstream, the $y$ axis is perpendicular (defined as transverse) to the flow direction and the pipe axis, and the $z$ axis lies along the axis of the pipe (defined as spanwise).

The visualized flow patterns and the measured velocities on the vertical symmetric plane are presented to highlight the interactions occurring between the discharged jet and the oncoming as well as the penetration and spreading of the jet. For each flow setting, PIV measurements were conducted on the cross plane located at $z=0$.

The injection ducts are circular pipes whose diameter is equivalent to $d=10 \mathrm{~mm}$. The injection hole resulted from the intersection of the injection pipe with the wind tunnel is ellipse shaped with $d$ and $D=d /(\sin \alpha)$ as minor and major diameters, respectively.

\section{NUMERICAL STUDY}

Consider a circular round jet of a pollutant of diameter $d$ emitted through a wall with an ejection velocity $v_{0}$, and a temperature $T_{0}$. This jet is subjected to a transverse flow with a uniform velocity $u_{\infty}$ and a temperature $T_{\infty}$. The ratio between the two velocities, $R=v_{0} / u_{\infty}$ is obtained by varying $u_{\infty}$ whereas $v_{0}$ is maintained constant. Consideration is given to a steady, three-dimensional and turbulent flow. The equations describing this flow are obtained in a system of Cartesian coordinates, the origin of which are situated in the center of the section of ejection of the jet (Fig. 1). We have the following: Continuity:

$$
\frac{\partial \bar{\rho}}{\partial t}+\frac{\partial\left(\bar{\rho} \tilde{u}_{i}\right)}{\partial x_{i}}=0
$$

Momentum equations:

$$
\begin{aligned}
\frac{\partial\left(\bar{\rho} \tilde{u}_{i}\right)}{\partial t} & +\frac{\partial\left(\bar{\rho} \tilde{u}_{j} \tilde{u}_{i}\right)}{\partial x_{j}}=-\frac{\partial \bar{p}}{\partial x_{i}}+\frac{\partial}{\partial x_{j}}\left(\mu \frac{\partial \tilde{u}_{i}}{\partial x_{j}}-\overline{\rho u_{i}^{\prime \prime} u_{j}^{\prime \prime}}\right) \\
& +\left(\bar{\rho}_{\infty}-\bar{\rho}\right) g \delta_{i j}
\end{aligned}
$$

Energy equation:

$$
\frac{\partial\left(\bar{\rho} \tilde{u}_{j} \tilde{T}\right)}{\partial x_{j}}=\frac{\partial}{\partial x_{j}}\left[\left(\frac{\mu}{\operatorname{Pr}}+\frac{\mu_{t}}{\sigma_{t}}\right) \frac{\partial \tilde{T}}{\partial x_{j}}\right]
$$

Species equations:

$$
\frac{\partial\left(\bar{\rho} \tilde{u}_{j} \tilde{f}\right)}{\partial x_{j}}=\frac{\partial}{\partial x_{j}}\left[\left(\frac{\mu}{S c}+\frac{\mu_{t}}{\sigma_{f}}\right) \frac{\partial \tilde{f}}{\partial x_{j}}\right]
$$

The introduction of the fluctuating sizes makes this system open. Its closing requires the use of a turbulence model which makes it possible to obtain a number of equations equal to the number of unknown parameters.

Mahjoub it al. (2003) show that the three first-order models (the standard $k-\varepsilon$ model, the $k-\varepsilon$ RNG model, and the realizable $k-\varepsilon$ model) render identical results in the upstream and far-downstream regions of the jet. However, only the second-order model is shown to give good results in the exit region and in the trailing zone of the jet. Based on this last result, we choose this second-order closure model (also called Reynolds stress model) in this work. So the following equation is solved:

$$
\begin{aligned}
& \frac{\partial}{\partial x_{k}}\left(\bar{\rho} \tilde{u}_{k} \overline{u_{i}^{\prime \prime} u_{j}^{\prime \prime}}\right)=\frac{\partial}{\partial x_{k}} \mu \frac{\partial}{\partial x_{k}}\left(\overline{u_{i}^{\prime \prime} u_{j}^{\prime \prime}}\right) \\
& -\bar{\rho}\left[\overline{u_{i}^{\prime \prime} u_{k}^{\prime \prime}} \frac{\partial \tilde{u}_{j}}{\partial x_{k}}+\overline{u_{j}^{\prime \prime} u_{k}^{\prime \prime}} \frac{\partial \tilde{u}_{i}}{\partial x_{k}}\right]+D_{i j}^{T}+G_{i j}+\phi_{i j}+\varepsilon_{i j}
\end{aligned}
$$

where $\partial /\left(\partial x_{k}\right)\left(\bar{\rho} \tilde{u}_{k} \overline{u_{i}^{\prime \prime} u_{j}^{\prime \prime}}\right)=C_{i j} ; \partial /\left(\partial x_{k}\right) x \mu \partial /\left(\partial x_{k}\right)$ $\times\left(\overline{u_{i}^{\prime \prime} u_{j}^{\prime \prime}}\right)=D_{i j}^{L} ; \overline{u_{i}^{\prime \prime} u_{k}^{\prime \prime}}\left(\partial \tilde{u}_{j}\right) /\left(\partial x_{k}\right)+\overline{u_{j}^{\prime \prime} u_{k}^{\prime \prime}}\left(\partial \tilde{u}_{i}\right) /\left(\partial x_{k}\right)$ $=P_{i j}$.

$C_{i j}$ being the convective term. $D_{i j}^{L}, P_{i j}, D_{i j}^{T}, G_{i j}, \phi_{i j}$, $\varepsilon_{i j}$ are, respectively, the molecular diffusion, the stress production, the turbulent diffusion, the buoyancy production, the pressure strain, and the dissipation rate (Schieste, 1993).

Equations of turbulent kinetic energy $(k)$ and dissipation rate of kinetic energy $(\varepsilon)$ associated with the secondorder model are defined as

$$
\begin{aligned}
\frac{\partial\left(\bar{\rho} \tilde{u}_{j} k\right)}{\partial x_{j}}=\frac{\partial}{\partial x_{j}}\left[\frac{\left(\mu+\mu_{t}\right)}{\sigma_{k}} \frac{\partial k}{\partial x_{j}}\right]+\frac{1}{2}\left(P_{i i}+G_{i i}\right)-\bar{\rho} \varepsilon \\
\frac{\partial\left(\bar{\rho} \tilde{u}_{j} \varepsilon\right)}{\partial x_{j}}=\frac{\partial}{\partial x_{j}}\left[\frac{\left(\mu+\mu_{t}\right)}{\sigma_{\varepsilon}} \frac{\partial \varepsilon}{\partial x_{j}}\right]+C_{\varepsilon 1} \frac{1}{2} \frac{\varepsilon}{k} P_{i i} \\
-C_{\varepsilon 2} \frac{\bar{\rho} \varepsilon^{2}}{k}
\end{aligned}
$$

and

$$
\begin{gathered}
P_{i i}=2 \mu_{t}\left(\frac{\partial \tilde{u}_{i}}{\partial x_{i}}\right)^{2}, \\
G_{i i}=2 \beta g_{i} \frac{\mu_{t}}{\sigma_{t}} \frac{\partial \tilde{T}}{\partial x_{i}}, \\
\mu_{t}=C_{\mu} \bar{\rho} \frac{k^{2}}{\varepsilon}
\end{gathered}
$$

The used constants are defined in Table 1.

The boundary conditions associated with the above system of differential equations are summarized in Table 2 . 
TABLE 1: Constants of the second-order model

\begin{tabular}{|c|c|c|c|}
\hline$C_{\varepsilon 1}$ & $C_{\varepsilon 2}$ & $\sigma_{k}$ & $\sigma_{\varepsilon}$ \\
\hline 1.44 & 1.92 & 0.82 & 1.0 \\
\hline
\end{tabular}

\section{NUMERICAL PROCEDURE}

Several important problems arise from the numerical simulation of this last system of equations. The topology of the flow requires a very fine meshing in a great part of the domain. In order to describe exactly any temperature or mass fraction variations, particularly near the jet, we adopted a nonuniform meshing, strongly tightened near the jet (Fig. 3).

The numerical code uses the finite volume method, the solution of equations being based on the algorithm SIMPLER proposed by Patankar and Spalding (1972). For the obtained algebraic equations we use an implicit scheme. The convection terms are interpolated from the values at the center of every face of the control volume, with a second-order upwind scheme, and the discretization of the diffusion terms is made with a second-order finitedifferences method. The elimination method of Gauss associated with an under-relaxation technique is used to solve the resulted tridiagonal matrix. The iterative process is stopped when the convergence criterion defined as

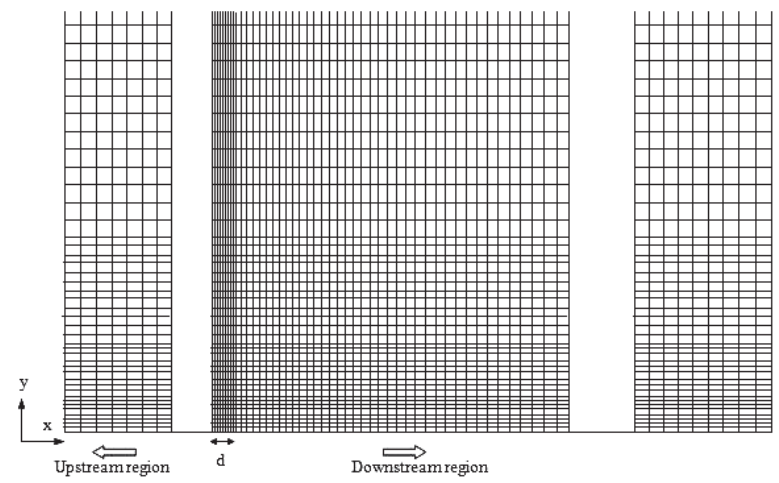

FIG. 3: Schematic presentation of the grid at the symmetry plane.

$$
\left|\frac{f^{m+1}-f^{m}}{f^{m+1}}\right| \leq 10^{-5}
$$

is satisfied. In Eq. (8), $f$ stands for velocity components, turbulent kinetic energy, and its dissipation rate, while $m$ is the iteration number.

\section{RESULTS AND DISCUSSION}

Figure 4 represents the evolution of an inclined jet characterized by a Reynolds number equal to 6000 (at rest flow)

TABLE 2: Boundary conditions

\begin{tabular}{|c|c|c|c|c|c|}
\hline Boundaries & Velocity & Temperature & Mass fraction & Kinetic energy & Rate of dissipation \\
\hline Jet hole & $\begin{array}{c}\tilde{u}=v_{0} \cos \alpha, \\
\tilde{v}=v_{0} \sin \alpha, \\
\tilde{w}=0\end{array}$ & $\tilde{T}=T_{0}$ & $\tilde{f}=f_{0}$ & $k_{0}=0^{-3} v_{0}^{2}$ & $\begin{array}{c}\varepsilon_{0}=k_{0}^{3 / 2} / 0.5 d \\
\text { Demuren and Rodi (1987) }\end{array}$ \\
\hline Crossflow & $\begin{array}{c}\tilde{u}=u_{\infty} \\
\tilde{v}=0 \\
\tilde{w}=0\end{array}$ & $\tilde{T}=T_{\infty}$ & $\tilde{f}=0$ & $k_{\infty}=0.0005 u_{\infty}^{2}$ & $\begin{array}{c}\varepsilon_{\infty}=k_{\infty}^{3 / 2} / 0.2 H_{T} \\
\text { Demuren and Rodi (1987) }\end{array}$ \\
\hline Ground & $\begin{array}{l}\tilde{u}=0, \\
\tilde{v}=0, \\
\tilde{w}=0\end{array}$ & $\frac{\partial \tilde{T}}{\partial y}=0$ & $\frac{\partial \tilde{f}}{\partial y}=0$ & $k=0$ & $\frac{\partial \varepsilon}{\partial y}=0$ \\
\hline $\begin{array}{l}\text { Other } \\
\text { boundaries } \\
\text { of the domain }\end{array}$ & $\begin{array}{l}\frac{\partial \tilde{u}}{\partial n}=0, \\
\frac{\partial \tilde{v}}{\partial n}=0, \\
\frac{\partial \tilde{w}}{\partial n}=0\end{array}$ & $\frac{\partial \tilde{T}}{\partial n}=0$ & $\frac{\partial \tilde{f}}{\partial n}=0$ & $\frac{\partial k}{\partial n}=0$ & $\frac{\partial \varepsilon}{\partial n}=0$ \\
\hline
\end{tabular}




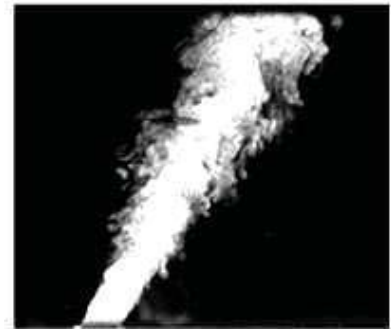

(i)

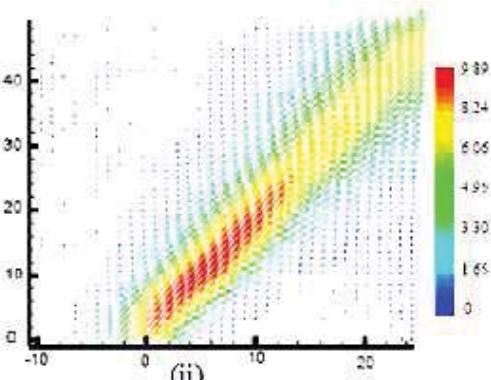

(ii)

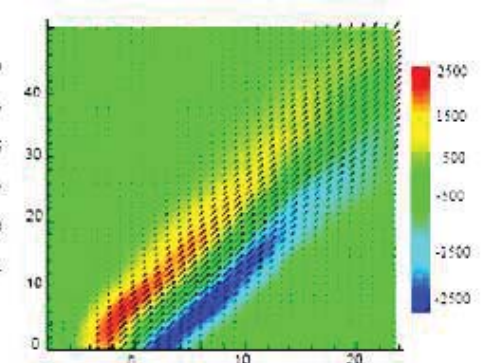

(iii)
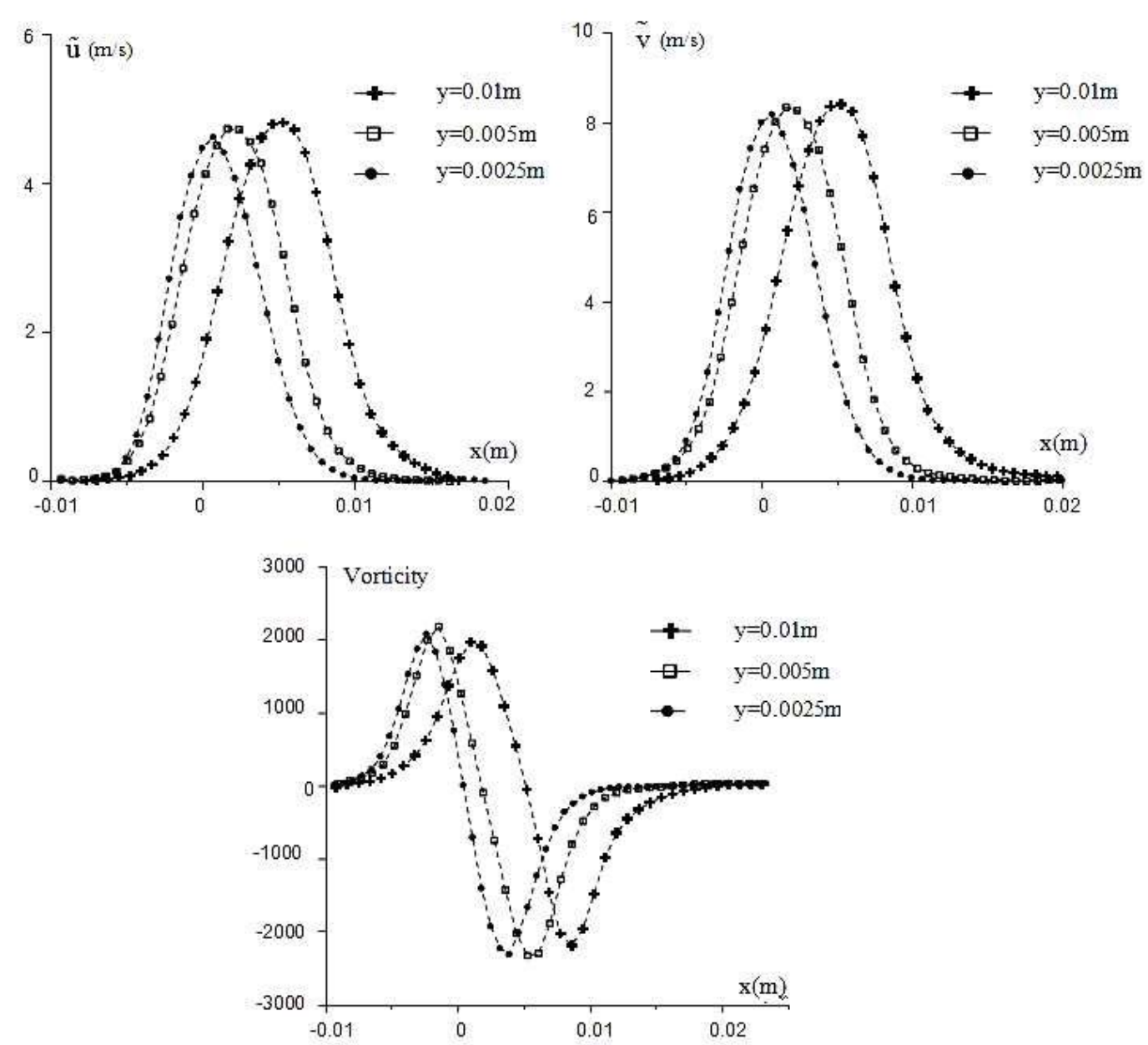

FIG. 4: (i) Mean velocity field, (ii) mean vorticity field, (iii) field structure for $\alpha=60^{\circ}, v_{0}=9 \mathrm{~m} \cdot \mathrm{s}^{-1}, u_{\infty}=0$ $\mathrm{m} \cdot \mathrm{s}^{-1}$.

and emerging from the pipe at $v_{0}=9 \mathrm{~m} \cdot \mathrm{s}^{-1}$. The figure presents also the contours of the vorticity along the center plane of the jet $(z=0)$. The jet fluid is deflected in the direction of the inclined jet. To these contours, we joined the longitudinal variation of the longitudinal and normal velocities components $(\tilde{u}$ and $\tilde{v}$ ) and the vorticity under an initial inclination angle equivalent to $\alpha=60^{\circ}$. The profiles are plotted on the symmetry plane $(z=0)$ and in the 
following vertical positions $y: y=1 / 4 d=2.5 \mathrm{~mm}, y=$ $d=5 \mathrm{~mm}$, and $y=d=10 \mathrm{~mm}$. Theses profiles show the development of the jet near its exit section in absence of a transverse flow. The velocity profiles have a symmetrical trend with respect to the vertical axis at $y=1 / 4 d$ (around the position $x=0$ ). Once the vertical coordinate increases, the potential core of the jet accuses some deformations. The deformation is quite obvious; it consists in the shifting of the center of symmetry downstream (from $x=0$ to $x \approx 6 \mathrm{~mm}$ ).

Figure 5 presents the evolution of a jet issuing at an angle of $60^{\circ}$, deflected in the direction of the crossflow. In the periphery of the jet, as the potential core of the jet ends as a cone, a mixing layer develops, slightly bent in the direction of the crossflow. The resulted flow field is quite complex. Accurate predictions of the flow have been difficult to obtain, particularly in the near-field of the injected jet. In fact, the flow field is characterized by a spectrum of vortical structures including the wake vortices and the shear layer vortices. A sharp velocity gradient is detected upstream of the jet while a "wake" region develops downstream of the jet. Vortices on the leeward edge of the jet are observed, and the highest velocity gradient is associated with these vortical structures.

Streamlines on the symmetry plane are also shown in Fig. 5, representing the path taken by the jet fluid as it interacts with the crossflow. Note that the jet is not symmetric about the mean streamline. The jet appears thinner on the upstream side of the streamline and thicker on the downstream side.

Figure 6 demonstrates the impact of both the jet streamwise inclination angle $\left(90^{\circ}\right.$ and $\left.60^{\circ}\right)$ and the blowing ratio $(R<1, R=1, R>1)$ on the flow behavior. The evolution of the jet structure while interacting with the mainstream is distinctly different under the two injection angles of $90^{\circ}$ and $60^{\circ}$. A significantly greater penetration and mixing of the jet with the crossflow is observed for the normally injected jet. The normal injection case indicates larger separation regions and leads to the reduction

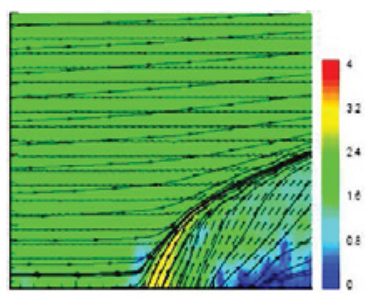

Streamlines field and velocity vectors

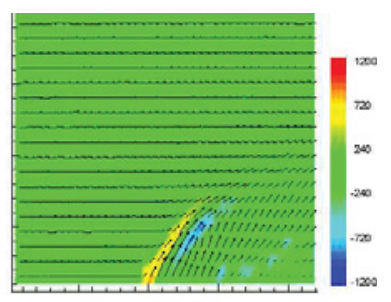

Mean vorticity field
FIG. 5: Various structures of an inclined jet.
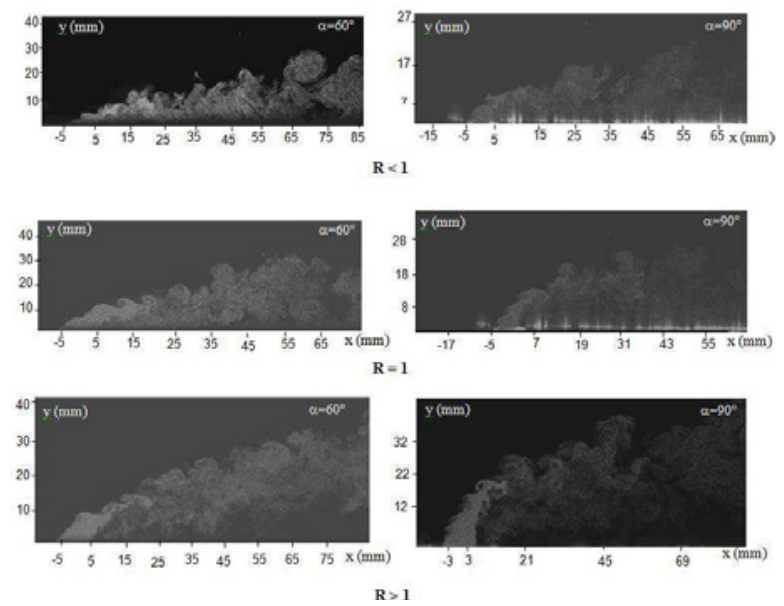

FIG. 6: Evolution of the flow field in the symmetry plane near the jet exit region for various velocity ratios.

of the wall shear stress. The normal injection of the jets is at the genesis of the observed greater penetration of the jet and the greater mixing between the jet and the mainstream. The blocking effect of the jet on the crossflow is significantly decreased due to the streamwise inclination. On the windward side of the jet exit no separation can be identified. The crossflow is only slightly deflected by the jet flow. The jet hardly penetrates into the crossflow and barely separates at the leeward edge of the hole. On the leeside of the jet exit the spanwise rollers originating from the trailing edge of the hole develop with a similar pattern as for the normal injection case. Their strength and the size of the affected domain, however, are smaller. Due to the reduced inclination angle the generation of vorticity is obviously reduced. The streamwise inclination then reduces the vertical jet velocity.

The confrontation of the calculated and measured results relatively to the longitudinal velocity component $\tilde{u}$ (Fig. 7) gives a satisfying matching along all the variation of the feature for the both cases $\left(\alpha=90^{\circ}, \alpha=60^{\circ}\right)$. The results processed with the RSM model are closer to the experimental data which suppose that the second-order model is more adequate for the description of the experiments (Mahjoub ït al., 2003). The results coincide and comfort the obtained validation.

In Fig. 8, we proceeded in the same way with the vertical velocity component $\tilde{v}$ by superposing the experimental and the numerical results. Here, we do not obtain a total matching as with the longitudinal velocity. Just for the case of $\alpha=90^{\circ}$, we note a slight lack of experimental points. According to the above-discussed confronta- 


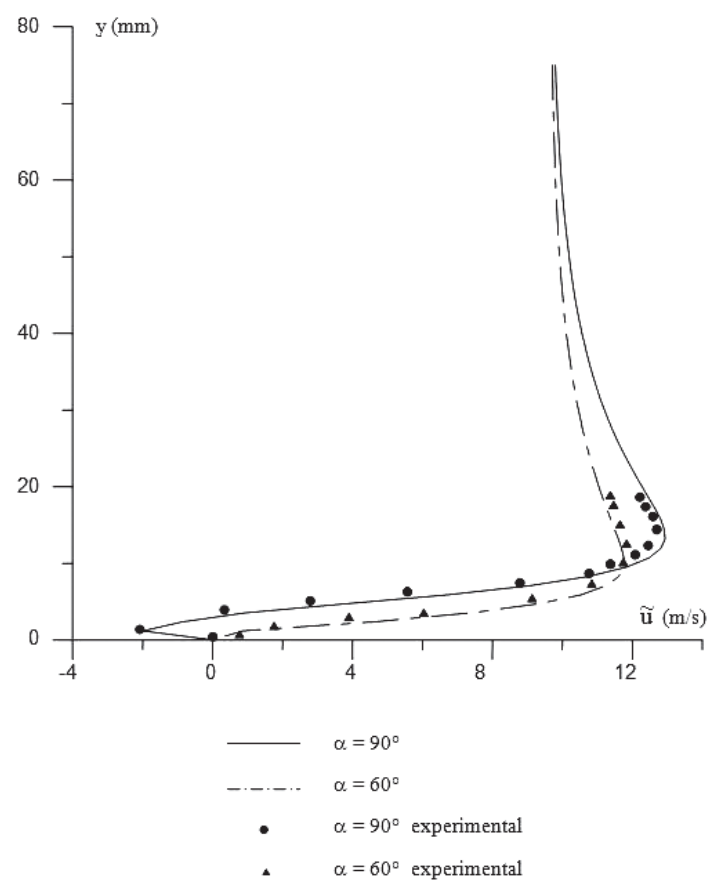

FIG. 7: Mean $\tilde{v}$-velocity profiles at the plane of symmetry $z=0$ and $R=2.3$ at $y=0.0025 \mathrm{~m}$ with different injection angle $\alpha$.

tions of the velocity components, and in accordance with the better description of the experiments with the RSM model, we can then conclude that our numerical modeling is acceptable and models well the experimental configuration of the inclined jet issuing within an oncoming crossflow.

We compared in Fig. 9 our numerical results to the experimental data of Crabb et al. (1981), obtained by hotwire anemometry. The evolution of the normal velocity $\tilde{v}$ is given in the symmetry plane $(z=0)$, for various injection angles $\left(\alpha=90^{\circ}, \alpha=60^{\circ}, \alpha=45^{\circ}, \alpha=30^{\circ}\right)$ and for a velocity ratio equivalent to $R=2.3$ for $y=0.0025 \mathrm{~m}$.

This figure shows the development of the jet near the ejection zone. For various injection angles, the profile of the velocity is uniform and symmetrical on the outlet side of the jet. The weaker the jet injection angle, the weaker the velocity. In fact, when the inclination angle is weak, the jet has the tendency to attach to the wall. It is well seen that for $\alpha=90^{\circ}$, the jet penetrates further in the transverse flow whereas for $\alpha=30^{\circ}$ the jet "is crushed" by the crossflow.

Figure 10 shows the variation of the mean longitudinal velocity $\tilde{u}$ according to the $y$ distance for various posi-

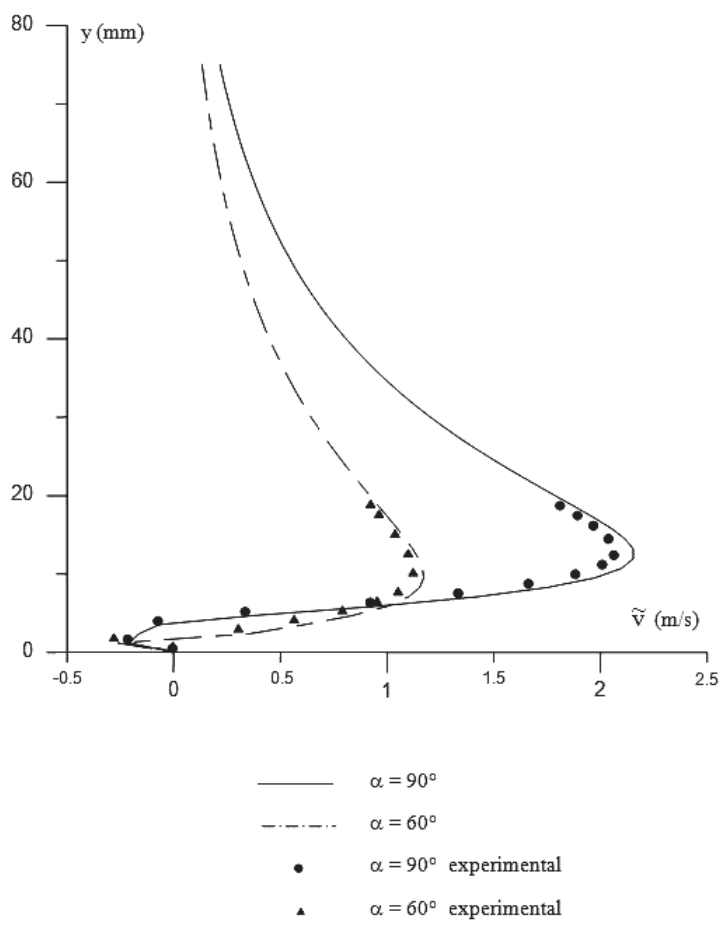

FIG. 8: Mean $\tilde{u}$-velocity profiles at the plane of symmetry $z=0$ for $R=0.5$.

tions $x$, in the symmetry plane $(z=0)$ : The profiles are obtained for a velocity ratio equal to $R=0.5$ and various injection angles $\alpha$ The first three profiles correspond to $x$ positions located at the top of the exit section and indicate a deceleration of the flow. The velocity increases tremendously for the weaker inclination at low and fixed values of $y$, i.e., near to the exit section of the jet. The last three profiles correspond to positions located downstream of the jet exit and indicate an acceleration of the flow in the wake region. The results are compared with those of Andreopoulos and Rodi (1984). For the position $x=0.02 \mathrm{~m}$, measurements of Bergeles et al. (1976) are also included within the comparison. The mean longitudinal velocity profiles show a peak which is considered as a locus of the jet trajectory. The peak's location is shifted downstream (increasing $y$ ) when the inclination angle decreases. Good agreement with the experiments is clearly indicated on this figure.

The study of the transverse component velocity allows determining the three-dimensional aspect of the considered flow. Figure 11 presents the transverse variation of this component at different longitudinal levels $(x=0.01$, $0.02,0.04,0.06,0.10 \mathrm{~m}$ ) at a given height $y=0.02 \mathrm{~m}$ 

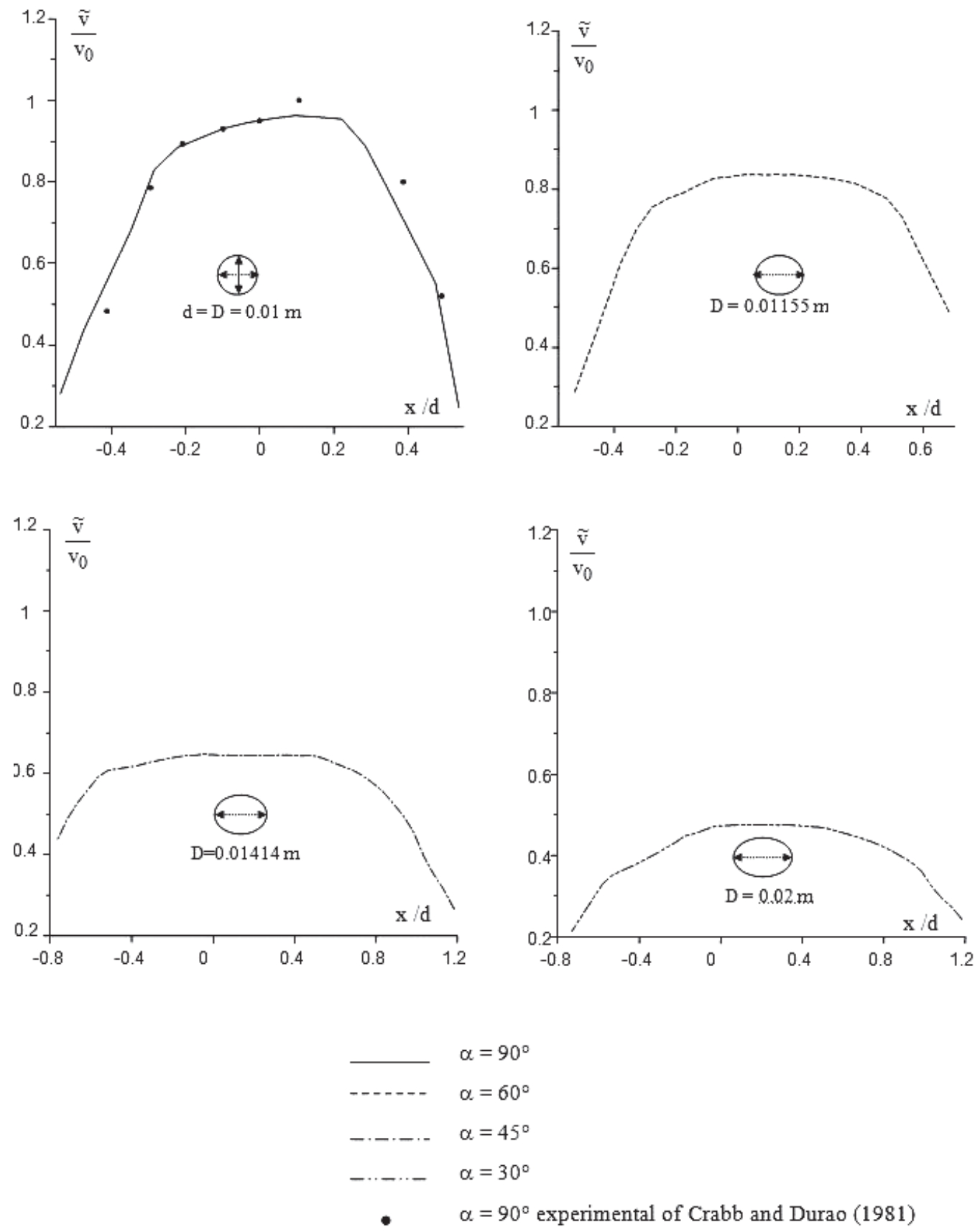

FIG. 9: Mean $\tilde{w}$-velocity profiles for $R=2$ and $\alpha=60^{\circ}$ at $y=0.02 \mathrm{~m}$.

and for a velocity ratio equivalent to $R=2$. The common feature between the plotted profiles is the alternation of a negative portion then of another positive. In fact, while emerging from the tube, the jet brings a dis- turbance to the transverse flow, which results in swirls into a horseshoe. These swirls turn on both sides of the jet column by intertwining; giving rise to a positive direction speed on the one hand and a negative direction 

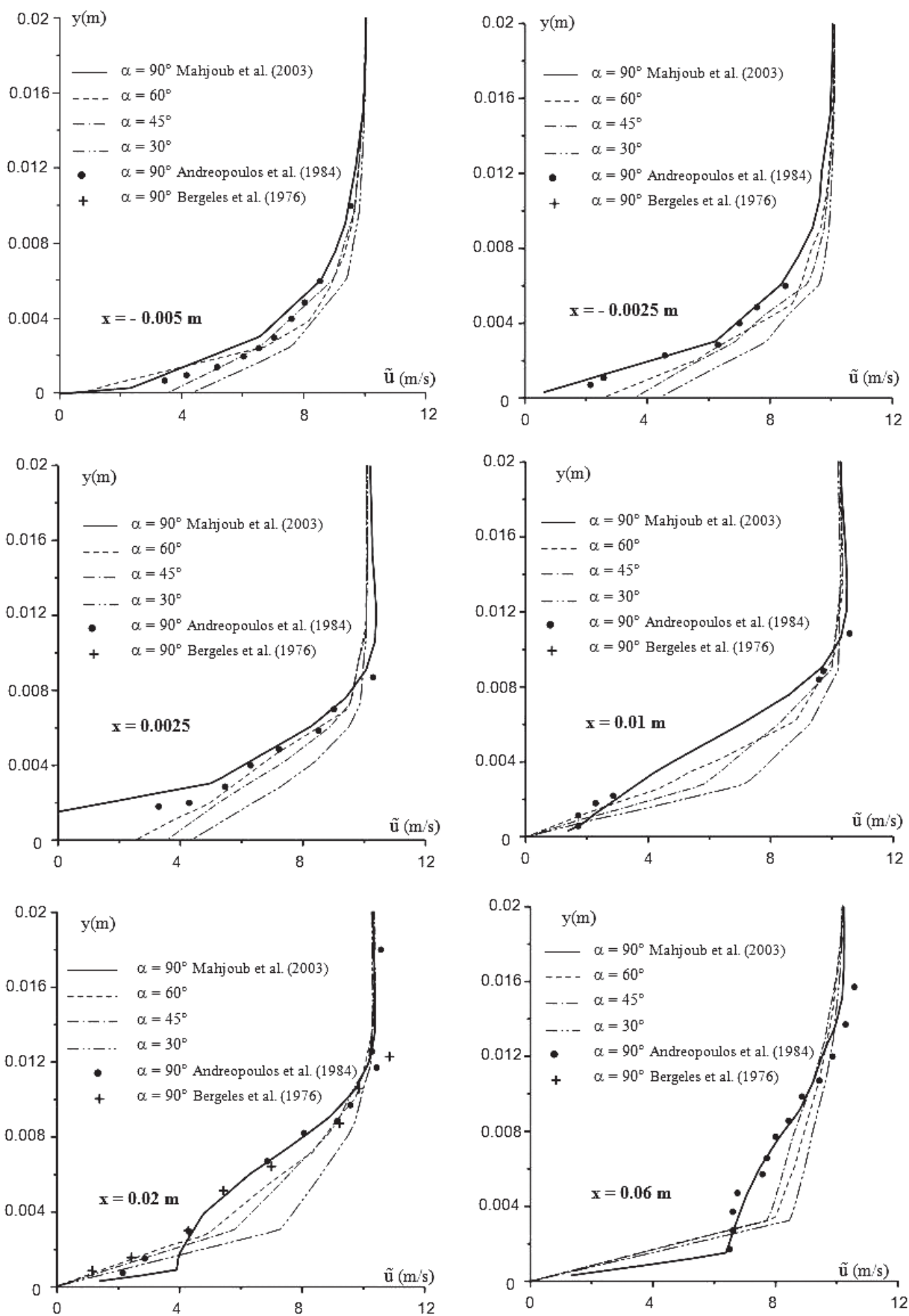

FIG. 10: Cross-sectional contours of the temperature at six planes and $R=5=60^{\circ}$.

speed on the other hand. We notice that the more we approach the edge downstream of the jet, the weaker the recorded gradient. We note also that the transverse com- ponent velocity decreases with $x$, which reveals that the more we move away from the jet, the less the training of the transverse current influences the flow. The 


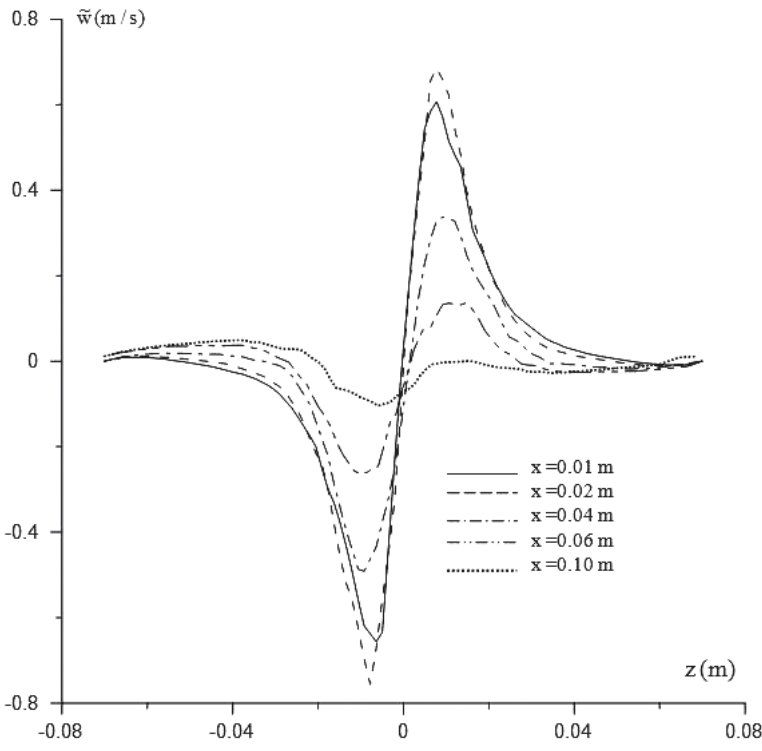

FIG. 11: Profiles of the temperature at $y=0.02 \mathrm{~m}$, for $R=5$ and $\alpha=60^{\circ}$.

mean transverse velocity is equal to zero in the symmetry plane.

In Fig. 12, we represented the evolution of the counterrotating vortex pair for six positions $(x=0.01,0.02,0.03$,
$0.04,0.05$ and $0.06 \mathrm{~m}$ ) under a constant velocity ratio equivalent to 5 . The average swirl issued from the jet is reorientated and packed up in a pair of swirls, which limits the surface of the jet under the effect of the transverse flow. Turbulence causes the training of the deviated jet and the majority of the transverse fluid in the wake region. This counter-rotating vortex pair is much more intense than the horseshoe swirl, leading to the formation of a kidney-shaped swirl (Moussa et al., 1977; Foss, 1980). The counter-rotating vortex pair is primarily a manifestation of the field of the mean flow induced by the initial impulse of the jet. It starts to take form in the near field, as indicated on the figure, and becomes dominant in the far field. This point was elucidated by Broadwell and Breidenthal (1984). The trajectory of the jet is conditioned by two elements: the movement tending to move the fluid away from the wall in the transverse direction, resulting from the initial impulse or the direction from the jet, and the bearing movement in the direction of the transverse flow (Coehlo and Hunt, 1989).

In this part, we considered a fume jet discharged at the temperature of $110^{\circ} \mathrm{C}$ within an air flow at a temperature equal to $18^{\circ} \mathrm{C}$. The fume is composed as follows: $20.9 \%$ carbon dioxide $\left(\mathrm{CO}_{2}\right), 76.9 \%$ nitrogen $\left(\mathrm{N}_{2}\right), 1.8 \%$ oxygen $\left(\mathrm{O}_{2}\right), 0.4 \%$ sulphur dioxide $\left(\mathrm{SO}_{2}\right)$.

Figure 13 represents the temperature evolution of an inclined jet $\left(\alpha=60^{\circ}\right)$ in the plan $y=0.02 \mathrm{~m}$ according

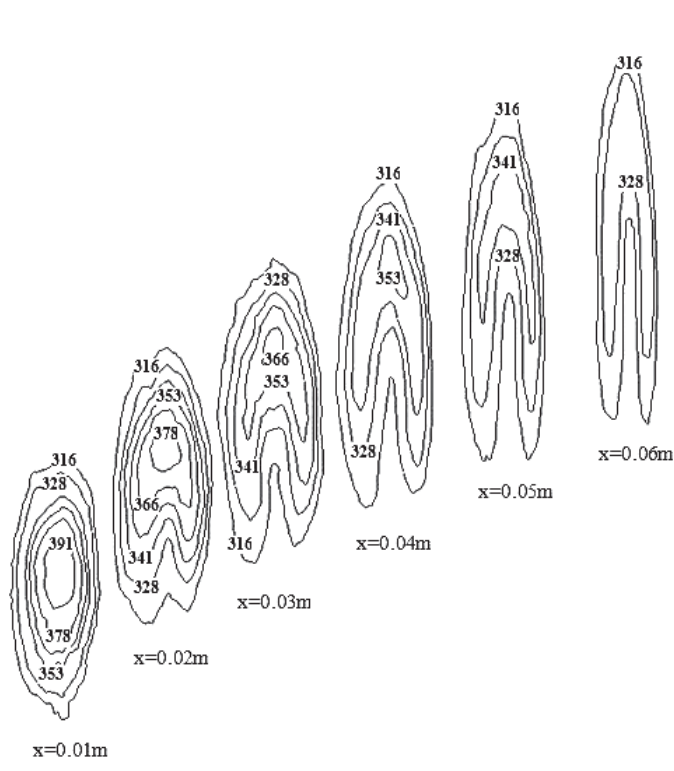

FIG. 12: Profiles of the temperature at $x=0.02 \mathrm{~m}$, for $R=5$ and $\alpha=60^{\circ}$.

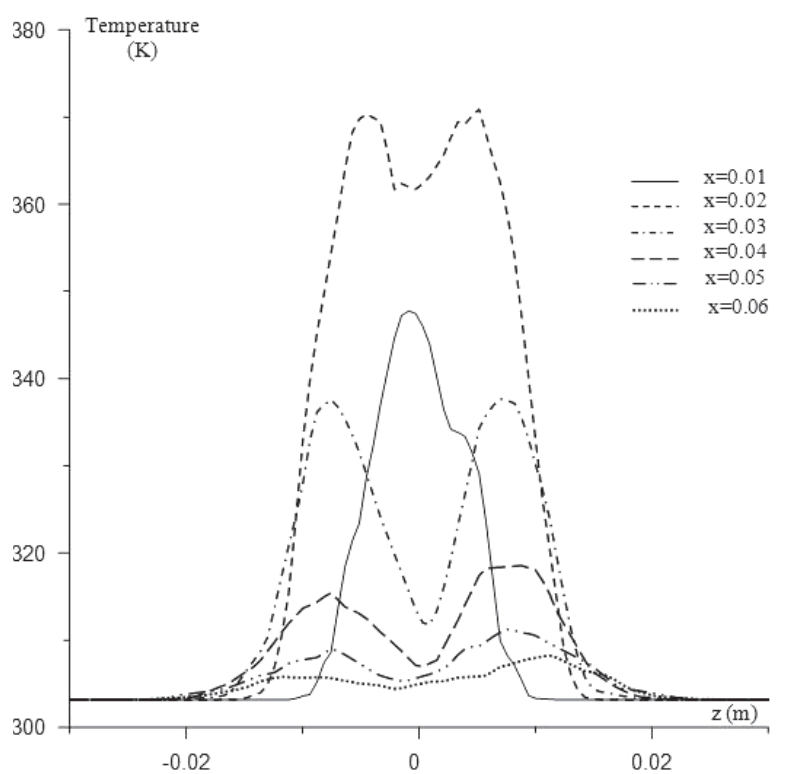

FIG. 13: Cross-sectional contours of the temperature at three planes and $R=2$. 
to distance $z$ for various emplacements $x$ and a velocity ratio $R=5$. For the positions $x=0.02,0.03$, 0.04, and $0.05 \mathrm{~m}$, the profiles present two peaks corresponding to the centers of the pair of swirls. The latter adopts the kidney shape caused by the longitudinal vortices. The presence of the swirls pair is remarkable for the position $x=0.02 \mathrm{~m}$. The two peaks attenuate and disappear for $x=0.06 \mathrm{~m}$. Beyond the latter the temperature decreases.

We traced similarly in Fig. 14 the evolution of the temperature but at a single position $x$ and at different levels $y$. The presence of the swirls pair is remarkable for the position $y=0.02 \mathrm{~m}$. At $y=0.01 \mathrm{~m}, y=0.015 \mathrm{~m}$, we note only a slight dissymmetry of the foyers. This asymmetrical character and its existence in spite of the configuration's symmetry and its cohabitation with the symmetry under certain conditions were the subject of certain studies, though not too many. Those of Smith and Mungal (1998) and Yuan et al. (1999) are included in that group since Yuan et al. (1999) showed the existence of asymmetry for the scalar instantaneous field of the concentration in various transverse plans: They noted it on contours of the transverse vorticity. Smith and Mungal (1998) suggested that this character can exist even for the realized structures and this, under certain conditions, applied to the flow.

In Fig. 15, we show the isotherms at three vertical levels $(y=0.005,0.01,0.02 \mathrm{~m})$ in the $x z$ plan under a veloc-

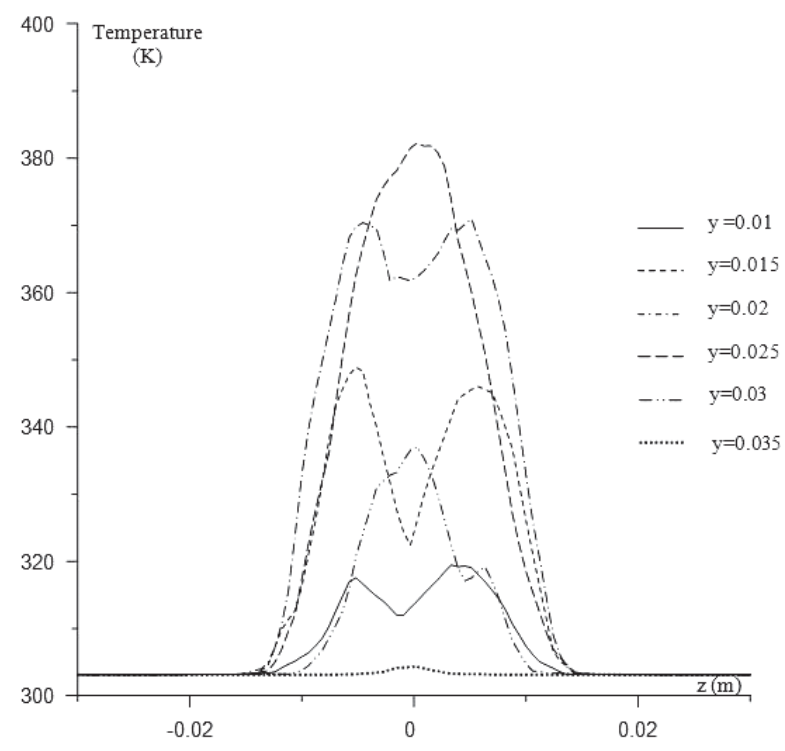

FIG. 14: Temperature contours at the plane of symmetry $z=0$ and $R=2$.
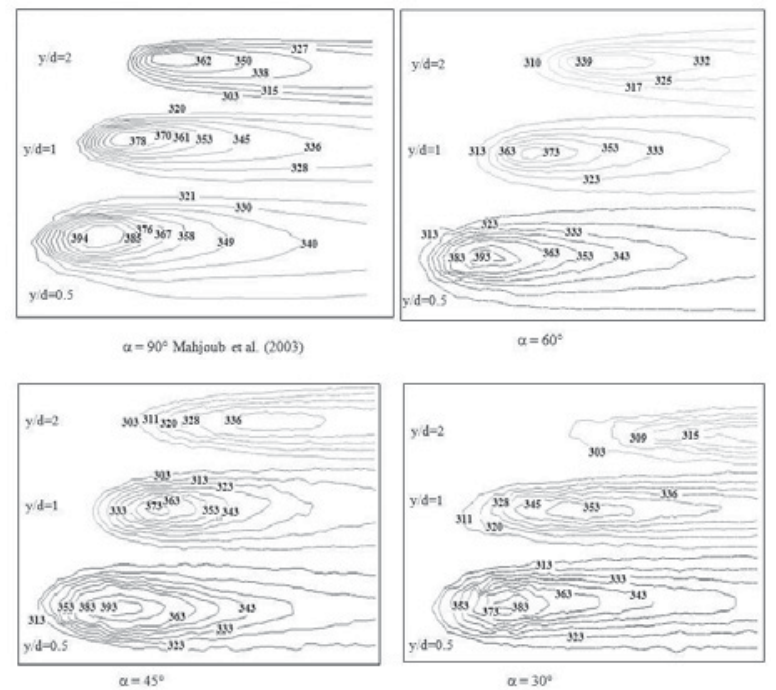

FIG. 15: Profiles of the temperature at $y=0.0025 \mathrm{~m}$ and $z=0$ for various velocity ratios $\left(60^{\circ}\right)$.

ity ratio equivalent to $R=2$. When the height $y$ increases, the maximum of temperature is shifted far downstream of the jet. The curve created by the transverse flow reveals a lateral bending of the jet, on both sides, caused by the three-dimensional flow. The dispersion of the temperature is faster in the three-dimensional case than it would be in a two-dimensional case because of the additional mixture induced by the transverse swirls. The temperature peak is more obvious at higher levels (higher $y$ ) and at larger inclinations $\alpha$.

In Fig. 16, we traced the isotherms in the central plan ( $z=0$ ), for a velocity ratio $R=2$ and for various injection angles $\alpha$ This figure shows the well-known Gaussian distribution and reveals a more significant wall reattachment under weaker injection angles.

In Fig. 17, we analyzed the influence of the velocity ratio $R(R=0.5,2,1)$ over the temperature distribution in the symmetry plane $z=0$, at a height equivalent to $y$ $=0.0025 \mathrm{~m}$ and under an inclination angle of $\alpha=60^{\circ}$. In the interval $-0.01 \mathrm{~m} \leq x \leq 0.01 \mathrm{~m}$, the pollutants' temperature attains the lowest peak under the lowest rate of the transverse flow $(R=0.5)$. In fact, under these circumstances, the flow is constrained against the injection wall. The mixing process is consequently enhanced near the injection wall, leading to a significant decrease in the pollutant's temperature. When we move far downstream, the most significant temperature peak is still obtained under the lowest injection rate ( $R=0.5$ ). In fact, under this same 

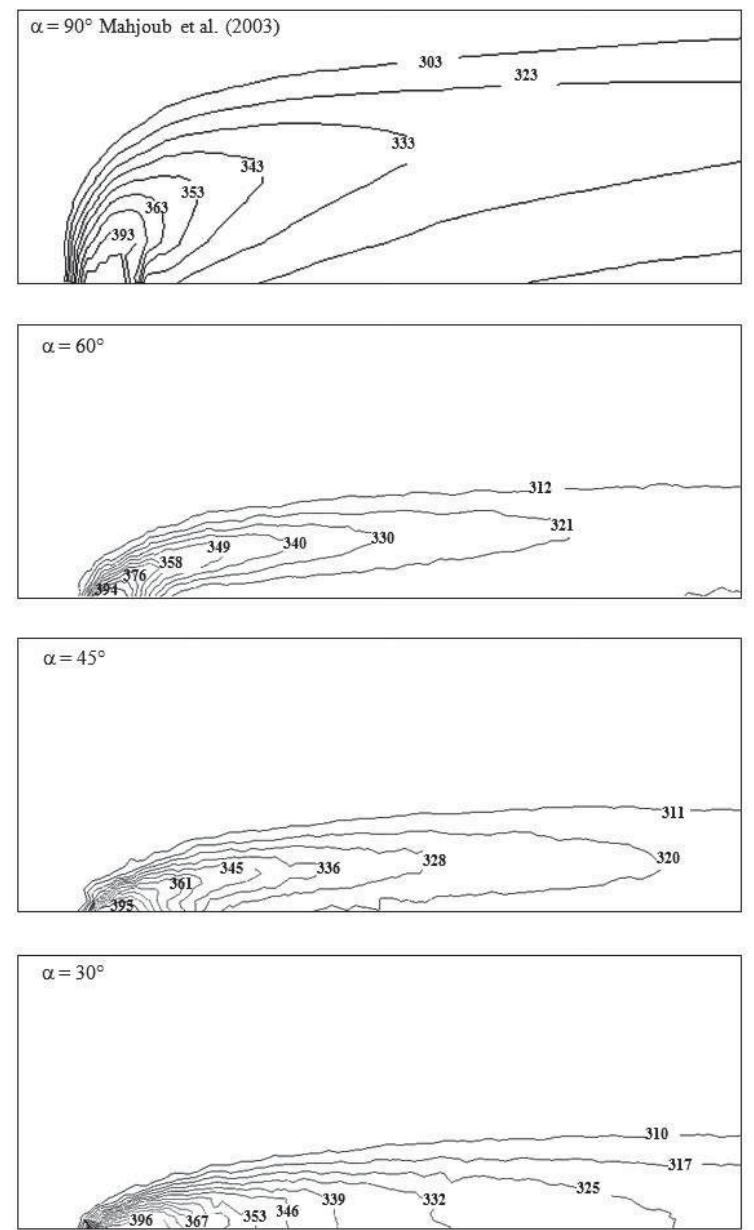

FIG. 16: Profiles of the temperature at $y=0.0025 \mathrm{~m}$ and $z=0$ for various inclined angles $(R=2)$.

rate, the flow is confined close to the injection wall and is oriented downstream. The jet plume is then less dispersed in the vertical direction than under higher ratios. The temperature of the jet plume is consequently further preserved justifying the higher registered temperature peak.

In Fig. 18, we analyzed the influence of the injection angle $\left(\alpha=30^{\circ}, 45^{\circ}, 60^{\circ}, 90^{\circ}\right)$ over the longitudinal distribution of the temperature in the symmetry plan $z=0$ at a height equivalent to $y=0.0025 \mathrm{~m}$ and under a velocity ratio equal to $R=2$. The temperature peak is attained over a whole stage, which is larger and slightly higher under weaker inclination angles. Far downstream, the highest thermal peak is also attained under the weakest initial inclinations $\left(\alpha=30^{\circ}\right)$ due to the confinement of the flow close to the wall.

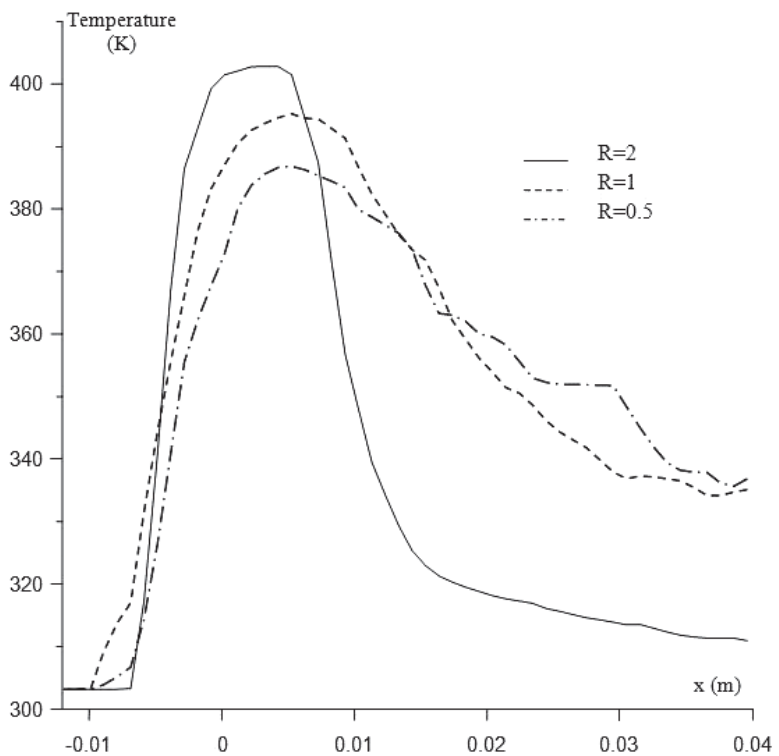

FIG. 17: $\mathrm{CO}_{2}$ mass fraction profiles at $y=0.0135 \mathrm{~m}$ for $R=2$.

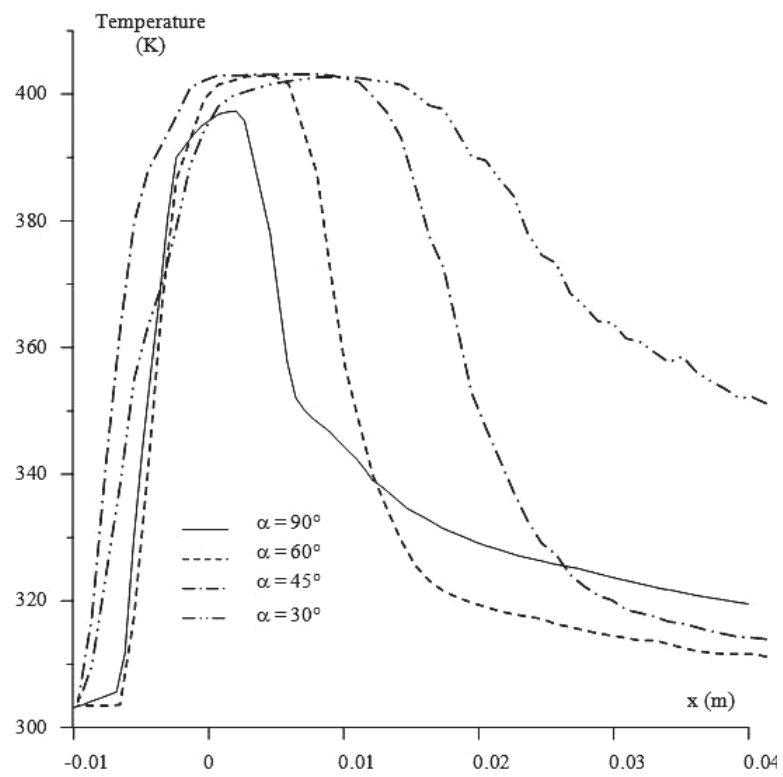

FIG. 18: Profiles of the temperature at $y=0.0025 \mathrm{~m}$ and $z=0$ for various inclined angles $(R=2)$.

Figure 19 represents the distribution of the mass fraction of carbon dioxide under $R=2$, at $y=0.0135 \mathrm{~m}$ and at various lateral locations ( $z=$ etc.). The pollutant's mass fraction decreases as one moves away from 
the jet exit section along the $z$ direction due to the further pronounced dispersion process along this direction. We also note that the peak of the pollutant's mass fraction is shifted further downstream as the jet is less straightened. This is due to the further longitudinal impulse provided by the weaker inclination angles, as further emphasized in Table 3. At $z=0.02 \mathrm{~m}$ and $z=0.025 \mathrm{~m}$, the dispersion process of the pollutant is already pronounced, justifying the weakness of the mass fraction of the carbon dioxide.

\section{CONCLUSION}

We have examined in this paper the dynamic and thermal fields as well as mass transfer generated by the interaction of an inclined jet with an oncoming crossflow. This study has been conducted both experimentally by means of the PIV technique and numerically by means of the finite volume method together with a turbulent closure model. The paper presents a three-dimensional experimental study of an inclined circular turbulent polluting jet issuing transversely into a uniform air stream. The dynamic field of a turbulent jet issuing transversely with velocity $v_{0}$ into a uniform air flow with velocity $u_{\infty}$ is examined as a function of the velocity ratio $R=v_{0} / u_{\infty}$. We also examined the structural behavior of the flow at different locations of the domain. The complexity of the observed vortical structures in the wake region as well as their interaction with the jet as it bends over after emission are discussed. Attention was also given to the Kelvin-Helmholtz vortex structures, established due to peripheral instabilities. We also studied the effect of the jet inclination angle on the various flow structures. The inclination angle of the jet is an important parameter that controls the development of the flow structures and the penetration and spreading of the jet. The results of a normal and an inclined jet in crossflow are performed to study the effect of the jet inclination on the penetration on the crossflow. In the perpendicular injection case a rather large separation region is found at the leading edge of the jet hole; whereas in the streamwise inclined injection cases no separation is observed. Compared with the normal injection case at the same blowing ratio, the streamwise inclination weakens the jet-crossflow interaction significantly. We also determined the distribution of the temperature and the mass fraction near the jet and downstream of it and examine the dispersion of a hot pollutant under the influence of the velocity ratio $R=v_{0} / u_{\infty}$. The obtained results show mainly that the temperature of the pollutants is low when the velocity of the transverse flow is high because, in this case, the crossflow prevails in front of the jet flow and high temperature gradients are observed near the exit section of the jet. Moreover, turbulence is sufficiently significant to allow a better dilution of pollutants. The different plotted profiles and contours of the temperature showed that increasing the initial streamwise inclination angle highly affects the temperature. This impact is expressed through the development of a further vertical, longitudinal, and lateral diffusion of the heat contained

TABLE 3: Position of maximal values of the mass fraction according to $x$ and $z$ directions

\begin{tabular}{|l|l|l|l|}
\hline$\alpha$ & $z$ & $x$ & Maximal mass fraction \\
\hline $90^{\circ}$ & 0.005 & 0.484848 & 0.122 \\
Mahjoub et al. (2003) & 0.01 & 0.767677 & 0.089 \\
& 0.015 & 1.9697 & 0.048 \\
& & 2.60606 & 0.021 \\
\hline $60^{\circ}$ & 0.005 & 0.0163636 & 0.122605 \\
& 0.01 & 0.0172727 & 0.0982105 \\
& 0.015 & 0.0236364 & 0.0425939 \\
& & 0.0161479 & 0.0518182 \\
\hline $45^{\circ}$ & 0.005 & 0.026061 & 0.185224 \\
& 0.01 & 0.030303 & 0.151212 \\
& 0.015 & 0.053636 & 0.0603705 \\
& & 0.108788 & 0.0195276 \\
\hline $30^{\circ}$ & 0.005 & 0.0515151 & 0.122965 \\
& 0.01 & 0.0706061 & 0.0806713 \\
& 0.015 & 0.11303 & 0.0323078 \\
\hline
\end{tabular}



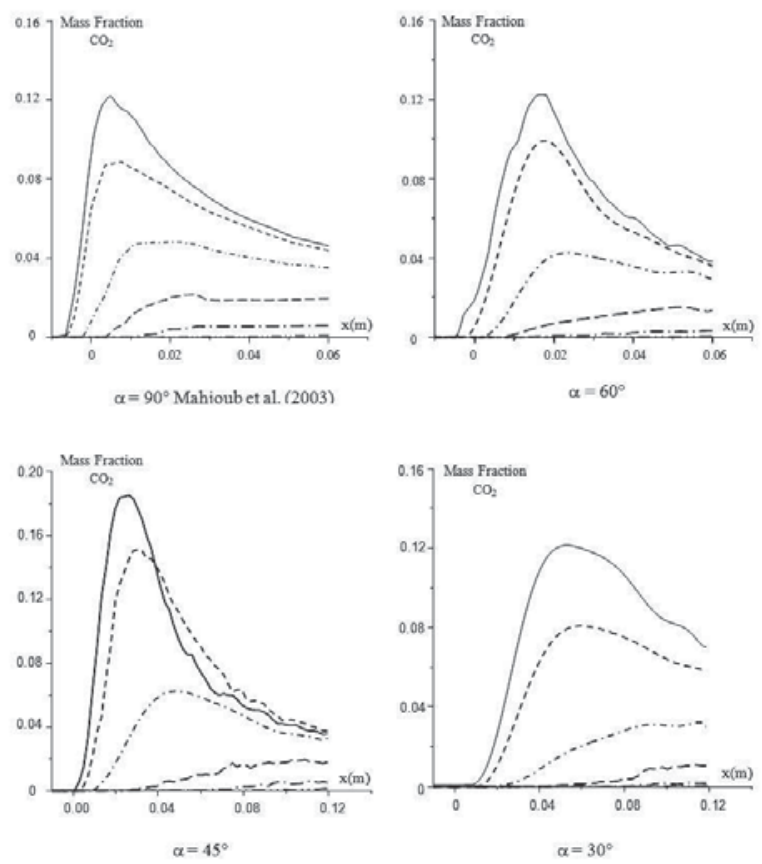

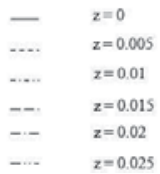

FIG. 19: $\mathrm{CO}_{2}$ mass fraction profiles at $y=0.0135 \mathrm{~m}$ for $R=2$.

within the potential core of the emitted jet. This diffusion allows a better mixing in these directions and results in a quicker homogenization of the global thermal field. The same trend is adopted by the mass fraction of the fume contained within the jet nozzle since it is lifted when its initial inclination is increased. That also limits the propagation of the fume in the longitudinal direction and then controls its dispersion on it. We can then conclude that the initial inclination angle highly affects both the thermal and mass transfer variations that result from the interaction of the emitted jet within the crossflow.

\section{REFERENCES}

Andreopoulos J. and Rodi, W., Experimental investigation of jets in a crossflow, J. Fluid Mech., vol. 138, pp. 93-127, 1984.

Andreopoulos, J., Measurements in a jet-pipe flow issuing perpendicularly into a cross stream, ASME J. Fluid Eng., vol. 104, pp. 493-499, 1982.
Azzi, A., Abidat, M., Jubran, B. A., and Theodoridis, G. S., Film cooling predictions of simple and compound angle injection from one and two staggered rows, Numer. Heat Transfer, Part A, vol. 40, pp. 273-294, 2001.

Bergeles, G., Gosman, A. D., and Launder, B. E., The near field character of a jet discharged normal to a main stream, ASME, J. Heat Transfer, vol. 98, p. 373, 1976.

Broadwell, J. E. and Breidenthal, R. E., Structure and mixing of a transverse jet in incompressible, flow, J. Fluid Mech., vol. 148, pp. 405-412, 1984.

Chu, V. H., Oblique turbulent jets in a crossflow, J. Eng. Mech., vol. 111, pp. 1343-1360, 1985.

Cortelezzi, L. and Karagozian, A. R., On the formation of the counter-rotating vortex pair in transverse jets. J. Fluid Mech., vol. 446, pp. 347-373, 2001.

Crabb, D., Durao, D. F. G, and Whitelaw, J. H., A round jet normal to a crossflow, Trans. ASME I: J. Fluids Eng., vol. 103, pp. 142-152, 1981.

Coehlo, S. L. V. and Hunt, J. C. R., The dynamics of the near field of strong jets in crossflows. J. Fluid Mech., vol. 200, pp. 95-120, 1989.

Demuren, A. O. and Rodi, W. Three dimensional numerical calculations of flow and plume spreading past cooling towers, $J$. Heat Transfer, vol. 109, pp. 113-119, 1987.

Donohue, J. M., McDaniel, Jr., J. C., and Haj-Hariri, H., Experimental and numerical study of swept ramp injection into a supersonic flowfield, AIAA J., vol. 32, no. 9, pp. 1860-1867, 1994.

Fischer, H. B., List, E. J., Koh, R. C. Y., Imberger, J., and Brooks, N. H., Mixing in Inland and Coastal Waters, New York: Academic Press, 1979.

Foss, J., Interaction region phenomena for the jet in a crossflow, Report no. SFB 80/E/161, University of Karlsruhe, 1980.

Fric T. F., Structure in the near field of the transverse jet, Ph.D. Thesis, California Institute of Technology, 1990.

Guo, Ting-ting, Li, Shao-hua, and Xu, Zhong, Flow characteristics of turbulent jets injected obliquely in crossflow, $\mathrm{J}$. $\mathrm{Hy}$ drodyn., vol. 16, no. 3, 2004.

Guo, X., Shröder, W., and Meinke, M., Large eddy simulations of film cooling flows, J. Comput. Fluids, 2006.

Jansson, L. S. and Davidson, L. Numerical simulation of inclined jets in a crossflow using a Reynolds stress model, in Simulation of turbulence, Turbulence III, 1994.

Jessen, W., Schroder, W., and Kalass, M., Evolution of jets effusing from inclined holes into crossflow, Int. J. Heat Fluid Flow, vol. 28, no. 6, pp. 1312-1326, 2007.

Jia, R., Sundén, B., Miron, P., and Léger, B., A numerical and experimental investigation of the slot film-cooling jet with various angles, J. Turbomachinery, vol. 127, no. 3, pp. 635645, 2005. 
Jirka, G, H., Ambrose, R. B., Martin, J. L., and Biswas, H., Technical Guidance Manual for Performing Waste Load Allocations, Book III: Estuaries, Washington, D.C.: United States Environmental Protection Agency, 1992.

Hasselbrink E. F. Transverse jets and jet flames: Structure, scaling and effects of heat release, Ph. D. Thesis, Stanford University, 1999.

Kelso R. M., Lim, T.T., and Perry, A. E., An experimental study of round jets in cross-flow, J. Fluid Mech., vol. 306, pp. 111144, 1996.

Lee, S. W., Lee, J. S., and Ro, S. T., Experimental study on the flow characteristics of streamwise inclined jets in crossflow on flat plate, J. Turbomachinery, vol. 116, no. 1, pp. 97-105, 1994.

Mahjoub, S. N., Mhiri, H., El Golli, S., Le Palec, G., and Bournot, Ph., Three-dimensional numerical calculations of a jet in an external crossflow: Application of dispersion of pollutants, J. Heat Transfer, Trans. ASME, vol. 125, pp. 510522, 2003.

Milanovic, I. M. and Zaman, K. B. M. Q., Highly inclined jet in cross-flow, in Proc. of 41st Aerospace Sciences Meeting and Exhibit, Reno, Nevada 6-9 January, 2003, AIAA-2003-0183, 2003.

Moussa, Z. M., Trischka, J. W., and Eskinazi, S., The near field in the mixing of a round jet with a cross-stream, J. Fluid Mech., vol. 80, pp. 49-80, 1977.

Nakabe, K., Yamamoto, Y., Fornalik, E., Chen, W., and Suzuki, K., Visualisations of obliquely discharged jet flows and their heat transfer enhancement regions, in Proc. of 8th Int. Symp. on Flow Visualization (CD-ROM, ISBN 09533991 0 9), 1998.

Norris, L. H. and Reynolds, W. C., Turbulent Channel Flow with a Moving Wavy Boundary, Report no. FM-10, Department of Mechanical Engineering, Stanford University, 1975.

Patankar, S. V. and Spalding, D. B., A calculation procedure for heat, mass and momentum transfer in three dimensional parabolic flows, Int. J. Heat Mass Transfer, vol. 15, pp. 17871806, 1972.

Rivero, A., Ferré, J.A., and Giralt, F., Organized motions in a jet in crossflow, J. Fluid Mech., vol. 444, pp. 117-149, 2001.

Roberts, P. J. W., Sea outfalls, in Environmental Hydraulics, edited by Hager, W. H. and Singh, V. P., Water Science and Technology Library series, Dordrecht: Kluwer Academic, Chap 3: pp. 65-109,1996.

Santiago, J. G., and Dutton, J. C . Velocity measurements of a jet injected into a supersonic crossflow, J. Propulsion Power, vol. 13, no. 2, pp. 264-273, 1997.

Schieste, Roland, Modélisation et simulation des écoulements turbulents, Paris: Hermès, 1993.

Smith, S. H. and Mungal, M. G., Mixing, structure and scaling of the jet in crossflow, J. Fluid Mech., vol. 357, pp. 83-122, 1998.

Taslim, M. E. and Ugarte, S., Discharge coefficient measurement for flow through compound-angle conical holes with cross-flow, Int. J. Rotating Machinery, vol. 10, pp. 145-153, 2004.

Taylor, P. and Watkins, D. J., An investigation of inclined jets in a crosswind, in AGARD Fluid Dynamics Panel Symposium on Fluid Dynamics of Jets with Applications to V/STOL, SEE N82-23150 14-01, 1982.

Yang, Y. T. and Wang, Y. X. Three-dimensional numerical simulation of an inclined jet with crossflow, Int. J. Heat Mass Transfer, vol. 48, pp. 4019-4027, 2005.

Yuan, L., Street, R. L., and Ferziger, J. H., Large-eddy simulations of a round jet in crossflow, J. Fluid Mech., vol. 379, pp. 71-104, 1999.

Wegner, B., Huai, Y., and Sadiki, A., Comparative study of turbulent mixing in jet in crossflow configurations using LES, Int. J. Heat Fluid Flow, vol. 25, pp. 767-775, 2004.

Zhang, X. and Collins, M. W., Measurements of a longitudinal vortex generated by a rectangular jet in a turbulent boundary layer, Phys. Fluids, vol. 9, no. 6, pp. 1665-1673, 1997. 